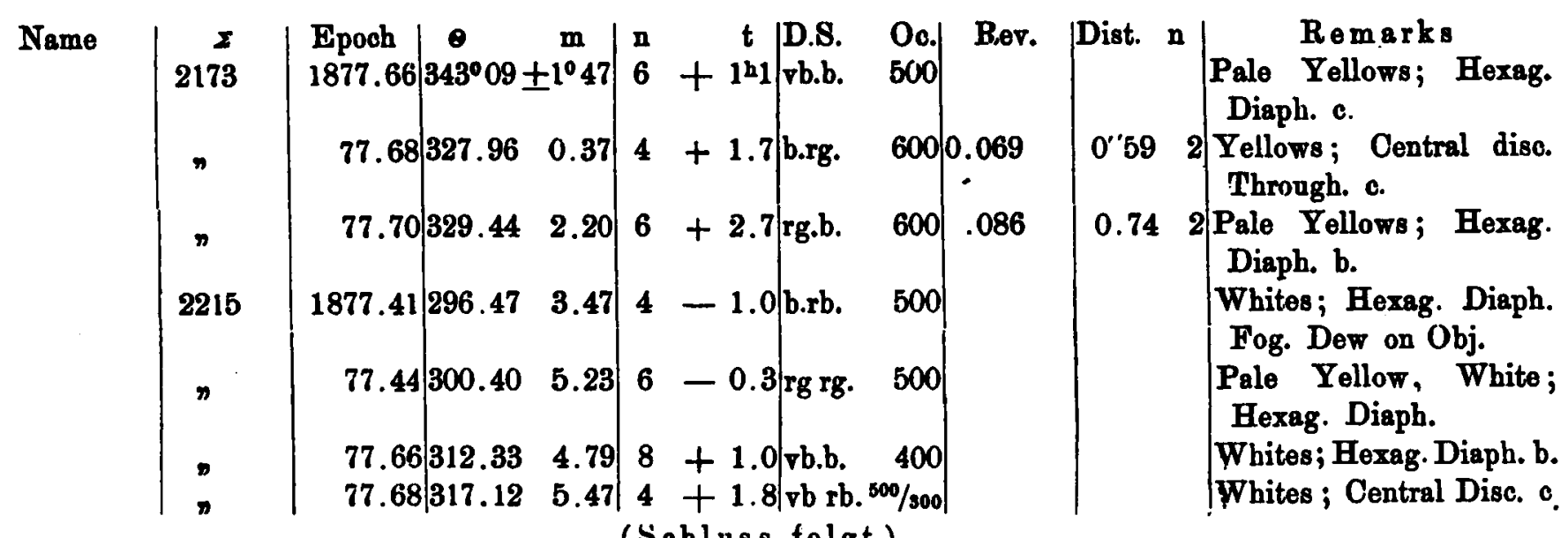

\title{
On the Correction of Approximate Double-Star Orbits.
}

For correcting orbits of double stars, those methods are preferable to the rigorous ones, that allow the discussion of the observations to go hand in hand with the derivation of the elements. - It is highly unsatisfactory to assume the relative weight of the observations proportional to the number of nights on which the ob: ject was measured. It might seem, as if I had fallen into the opposite error by entirely disregarding the number of measures, but I generally determine the normalplaces graphically, and then most weight is allowed to the best observations. The number of observations is besides of small importance, as they may have been made at short intervals. I assume the weight of the different observers' measures according to the respect 1 entertain for their ability and the means at their disposal, - being however fully aware that much knowledge remains to be gained from more extensive discussion of the observations.

I have computed two tables, which I fiad useful for solving Kepler's problem. The first gives, for every tenth of the eccentricity, the mean anomaly approximately with the true anomaly as argument. The second gives approximately the true with argument mean anomaly. They will be inserted in forthcoming Tables of mine with four decimals containing both logarithms and anti-logarithms, logarithmic-trigonometrical functions for every tenth or hundredth of a degree, and also natural sec. and tang. -

With the approximate epoch period and eccentricity I compute true anomalies corresponding to a number of normalplaces and obtain by equations of condition corrections to be applied to the three other elements. With their new values I calculate from the given angles of position the true auomalies, and from these, with different values of the eccentricity, the mean anomalies. From each of the different series of mean anomalies $I$ next obtain the epoch and period, and by a consideration of the outstanding errors $I$ interpolate a more correct value of the eccentricity and thence obtain the epoch and period. This epoch and period is now successively altered, and with the last value of the eccentricity three series of true anomalies are computed, from each of which by equations of condition the other elements are determined by the method of least squares. From the then outsstanding errors the differential coefficients of the angles with respect to the epoch and period are computed. These two elements are then obtained from equations of condition and therefrom the three geometrical elements. If the observations still are not satisfactorily represented, equations may easely be formed for correcting the three geometrical elements together with the eccentricity. This will generally suffice, and if a greater accuracy is considered attainable by an elliptic orbit, the problem may now be considered mature for the use of equations of condition obtained by differentiation. - New orbits of $\mu^{2}$ Boötis and $\omega$ Leonis were obtained by methods similar to this, through somewhat shorter.

Markree-Observatory 1878 April 26.

W. Doberck. 